\title{
Analysis of an Advanced Compounding Strategy based on Reactive Power Flow Measurement in the Medium Voltage Network
}

\author{
P. Franz ${ }^{1}$, G. Stapff ${ }^{1}$, I. Talavera ${ }^{1}$ and J. Hanson ${ }^{1}$ \\ ${ }^{1}$ Department of Electrical Power Supply with Integration of Renewable Energies \\ Technische Universität Darmstadt \\ Landgraf-Georg-Straße 4, 64283 Darmstadt (Germany)
}

\begin{abstract}
As well known, the increased share of renewable energies has led to new challenges for the electrical power system in Germany. Since the majority of renewable generation units are installed in the lower voltage levels, problems of congestion and voltage deviation occur more often. Commonly, the Distribution System Operators in Germany take advantage of a reactive power support by decentralised energy resources to ensure static voltage stability. The drawback of the support of reactive power for static voltage stability is the increasing demand for inductive reactive power by the distribution network which has to be compensated by the connected high voltage network. Besides, the power losses rise as well because of the additional reactive power flows. Therefore, other methods for voltage regulation like the use of compounding strategies at transformer stations could be advantageous to prevent the violation of voltage limits. This paper compares four different strategies of controlling the on-load tap-changer of the HV/MV-transformers in distribution networks and the effects on voltage deviation and power losses. A special focus is given to an advanced compounding strategy which adapts the control profiles of the compounding strategy dependent on the reactive power flow at the transformer as a second input parameter.
\end{abstract}

\section{Key words}

Compounding, Distribution Grids, On-Load Tap-Changer, Static Voltage Stability, Wide Area Voltage Control

\section{Introduction}

The increasing share of renewable energies $(23,4 \%$ of the total electricity generation [1]) in Germany has changed the previous system of network control. Due to the fact that most renewable energies are connected to the medium (MV) and low voltage (LV) levels, especially Distribution System Operators (DSOs) are affected by this penetration. Since distribution grids have not been designed to host a high amount of Dispersed Energy Resources (DERs), problems of network congestion and voltage deviations exceeding the voltage limits of the technical guidelines occur more often. A popular method in Germany is the use of inductive reactive power consumption by these generation units to solve voltage problems caused by a high power feed-in of DERs. This is in accordance with the technical standards which demand a contribution to static voltage stability by operating DERs with a certain power factor (depending on requirements by the DSO). In table 1 the parameters of German technical guidelines for the connection of DERs to LV- and MV-networks are shown.

Tab. 1: Demanded reactive power support of DERs by the German guidelines in the MV- and LV-networks [2], [3]

\begin{tabular}{|l|c|c|}
\hline & $\begin{array}{c}\text { Rated power } \\
\boldsymbol{S}_{\mathrm{a}, \max } \text { of DER }\end{array}$ & $\begin{array}{c}\text { Required power } \\
\text { factor } \cos \varphi\end{array}$ \\
\hline $\begin{array}{l}\text { BDEW-guideline } \\
\text { (MV-network) }\end{array}$ & all & $\begin{array}{c}0,95_{\text {underexcited }} \\
\leq \cos \varphi \leq \\
0,95_{\text {overexcited }}\end{array}$ \\
\hline & $\leq 13,8 \mathrm{kVA}$ & $\begin{array}{c}0,95_{\text {underexcited }} \\
\leq \cos \varphi \leq \\
0,95_{\text {overexcited }}\end{array}$ \\
VDE 4105 \\
(LV-network) & & $0,90_{\text {underexcited }}$ \\
& $\geq 13,8 \mathrm{kVA}$ & $\leq \cos \varphi \leq$ \\
& & $0,90_{\text {overexcited }}$ \\
\hline
\end{tabular}

In weak distribution networks an extensive use of reactive power support by DERs has normally the drawback, that the additional reactive power flow can cause overload of grid equipment, especially in times of maximum feed-in of DERs when the network is already close to its capacity limits. This increasing demand of distributions networks for reactive power has also to be compensated elsewhere which becomes more and more difficult in times of a decreasing number of big conventional power plants in the German transmission networks. Moreover, the additional reactive power flows increase the power losses in the networks.

Therefore, it can be advantageous to use the existing capacities of HV/MV-transformers for voltage control. This can help to improve the reactive power balance of a MV-network compared to an exclusive use of reactive power support for the reduction of voltage deviations. The operation of the network at a slightly higher voltage level (within the statutory voltage limits) reduces the power losses. But it has to be considered that centralised methods for voltage control often only work in networks with a more or less homogeneous character of the feeders (similar line length and same ratio of load/generation). Otherwise combinations of different methods, like a combined use of a flexible control of the on-load tap-changer (OLTC) and the reactive power support of DERs, can be advantageous to avoid voltage violations [4]. 


\section{Control methods for the tap changer}

In Germany nearly every HV/MV-transformer is equipped with an OLTC. The voltage at the secondary side of the transformer has to be adjusted in such a way that the voltage quality at the point of common coupling of the end-consumers fulfils the minimum requirements of the European Standard EN 50160 [7]. For the static voltage stability this standard permits a maximum deviation from the nominal voltage in a bandwidth of $\pm 10 \%$ of $U_{\mathrm{n}}$. The technical guidelines in Germany advise a margin for voltage raises because of DERs of $2 \%$ in the MV-level (BDEW [2]) and $3 \%$ in the LV-level (VDE 4105 [3]). For the voltage decrease because of loads a bandwidth of $5 \%$ for each voltage level is reserved, which is according to the typical criteria for network design in the past [8]. In figure 1 a possible partition of the permitted voltage band is shown. For the OLTC of the HV/MV-transformer a deadband of $\pm 1,5 \%$ of $U_{\mathrm{n}}$ is assumed. At maximum load the voltage drop over the impedance of the local power transformer can be up to a maximum of $2 \%$ of $U_{\mathrm{n}}$. Since LV-networks typically have inductive character, voltage increases over the impedance of the transformer with their high $X / R$-ratios normally do not occur [9].

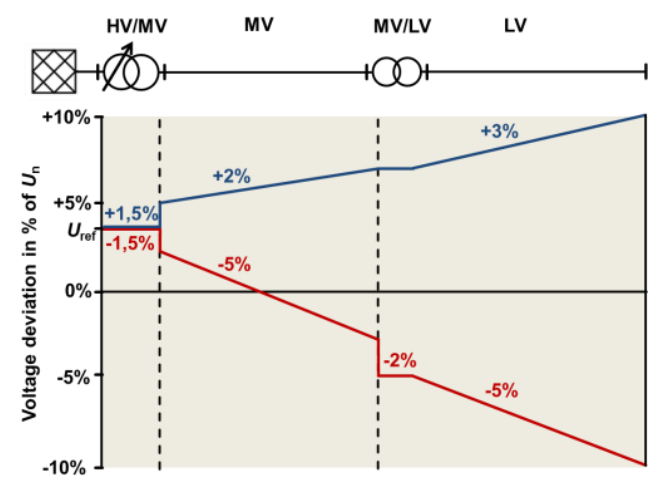

Figure 1: General partition of the voltage band

Because of the persistent increasing share of DERs, it becomes more and more challenging for the DSOs in Germany to keep the voltage within the limits, especially in rural grids. Since problems of voltage band violations often occur well before thermal overload gets critical, modern equipment like voltage-regulated distribution transformers or innovative methods (compounding) can help to avoid grid expansion by a better utilisation of the permitted voltage margin. An implementation of voltageregulated distribution transformers raises the scope by a decoupling of the LV-level. In this paper an improved utilisation of the static voltage limits by alternative control methods of the HV/MV-transformer is analysed.

If all maximum voltage deviations at critical nodes in a MV-network would be perfectly known, the regulation bandwidth for the reference voltage $U_{\text {ref }}$ on the secondary side of the transformer could be expanded. The margin of the deadband for the OLTC has still to be considered (in this case $\pm 1,5 \%$ of $U_{\mathrm{n}}$ ), but the usable bandwidth for $U_{\text {ref }}$ could be increased in the ideal case to $98,5 \%-105,5 \%$. The considered partition of the voltage band (figure 2 ) is based on guidelines and has not the character of a defined standard. Depending on network topology and planning principles the DSOs can vary these boundary values [8]. Possible voltage rises in the MV-level by power flows coming from the LV-network are e.g. not included in the partition of the voltage band in figure 1 and 2 .

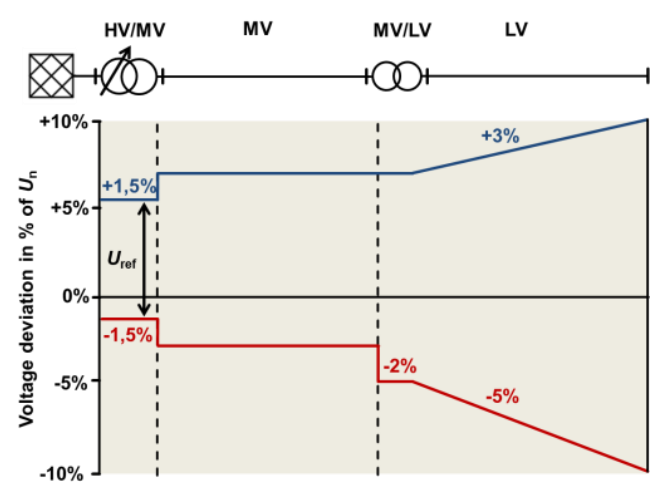

Figure 2: Increased control bandwidth by control methods

For the network simulations presented in this paper, the specific division of the voltage band is used to determine the control parameters of the OLTC and as a basis for the comparison of different control methods. In the following four different methods for the control of the OLTC are analysed and compared:

- Fixed reference voltage

- Wide area voltage control

- Static compounding

- Advanced compounding by $Q$-measurement

\section{A. Fixed reference voltage}

$\mathrm{Up}$ to now the tap-changer of a HV/MV-transformer in a network is controlled to keep a fixed reference voltage at the secondary side of the transformer. This is a sufficient method to distribute electricity to the consumers in the subordinate network levels and to host a small amount of DERs. The regulation is done by adjusting the tap position of the tap-changer depending on the difference between the measured voltage at the busbar and a desired reference voltage $U_{\text {ref. }}$. The reference voltage $U_{\text {ref }}$ is normally set to a value between 102 to $105 \%$ of $U_{\mathrm{n}}$. Because of the almost constant voltage at the busbar, this control method has a lower potential for the integration of further DERs than other control methods presented in the following. For a comparison of the results a fixed set point $U_{\text {ref }}$ of $103,5 \%$ is consistently used.

Since a mechanical OLTC can only adapt the voltage ratio stepwise, a tolerance margin around the reference voltage (deadband) is needed to avoid continuous switching. In practical operation the deadband is set symmetrical to $U_{\text {ref }}$ from $\pm 1 \%$ to $\pm 3 \%$. Smaller tolerance margins reduce the maximum voltage deviations in a grid with the drawback of an increasing number of tap changes which results in a faster wear of the contacts of the OLTC [10]. A tap change is performed when the measured voltage on the secondary side of the transformer is out of the defined deadband to bring the voltage back within the band [11]. To increase lifetime of the transformer and to eliminate unnecessary tap changes caused by short-term fluctuations a time delay for the switching operations is needed. A tap change is not directly performed when the measured voltage exceeds the deadband, but only if the period of voltage deviation is long enough (depending on the height of voltage deviation and typically set to about $10 \mathrm{~s}$ to $400 \mathrm{~s}[6,12])$. When a tap change is performed flicker problems can occur as well, which depend on the step size and the number of tap changes in a time interval. Therefore, it has to be ensured that the time intervals between switching operations are not too short and step sizes are not too high [13]. 


\section{B. Wide Area Voltage Control}

In the concept of a wide area voltage control (WAVC) the tap changer of the transformer has a remote control by measured voltage values at certain critical nodes in the distribution network. Thereby the most critical voltage deviations are exactly known and not only the voltage value at the busbar which can be affected by consumers or generation units connected directly to it. If in times of high generation the measured voltages at the selected nodes rise higher than the permitted voltage limit, the reference voltage at the secondary side of the transformer is reduced and a tap change is initiated by the OLTC. The same applies in the other direction when the voltage drops below the set limit. If all critical nodes in a network are measured, commonly at the end of the feeders, probably more DERs can be hosted in the MV-network. Another benefit of a WAVC is that the effects of a tap change on the voltage can be directly analysed. To increase lifetime of the OLTC again a deadband and time delay are needed. If free voltage margins are remaining, it is advantageous to operate the network at a possibly high voltage level to reduce power losses. In our simulations a tap change is initiated when the voltage at one of the critical nodes remains at a value of more than $1,07 \%$ of $U_{\mathrm{n}}$ or drops at one measured node below the value of $1,045 \%$ of $U_{\mathrm{n}}$. The drawbacks of this control method are the investment costs for the measurement devices at every critical node and operational costs for ICT-infrastructure and data analysis. Less measurement devices save costs, in return possibly higher safety margins have to be taken into account.

\section{Static compounding}

Higher costs for the ICT-infrastructure and measurement devices could be avoided in many network topologies by the implementation of a compounding strategy to control the OLTC of the transformer. The static compounding strategy is defined as a load-dependent control of the reference voltage $U_{\text {ref }}$. Therefore, besides the voltage, the current flow at the transformer has to be measured as well. The basic idea is that the adjustment of $U_{\text {ref }}$ depends on the magnitude and direction of the active power flow through the transformer. By the help of these power flow values the voltage deviations at the critical nodes in the MV-network can be estimated and serve as a substitute for real measured voltages of a WAVC. The accuracy depends on network topology and distribution of load and generation. In relatively homogenous networks this allows good estimations of the deviations along the feeders and therefore compensation by adapting the reference voltage at the busbar [14]. Of course these estimations are not as accurate as direct measurements, but there is no need for an ICT-infrastructure and data transfer, and it has a lower error rate. Costs are limited on measurement of voltage and current flow at the transformer, which are normally measured anyway, and an upgrade of the controller for the OLTC. High installed powers of DERs connected directly or close to the busbar can have negative effects on the usability of a compounding strategy. These units influence the power flow at the transformer without changing the voltage deviations along the feeders [15]. In those grids the implementation of a compounding is not applicable. The same applies for highly heterogeneous networks, e.g. having DERs in only one feeder and industrial loads in another one. Considering the division of the voltage band of figure 2, $U_{\text {ref }}$ can be varied in homogenous networks in a bandwidth of $0,985 U_{\mathrm{n}}$ to $1,055 U_{\mathrm{n}}$ if the active power flow through the transformer is used as an input parameter. The degree of optimisation depends on network topology and the profile of the $U_{\text {ref }} / P_{\text {load }}$-curve of the compounding strategy. Since topology and ratio of load/generation can differ for every MV-network, the optimal profile has to be determined for each network individually (figure 3 ).

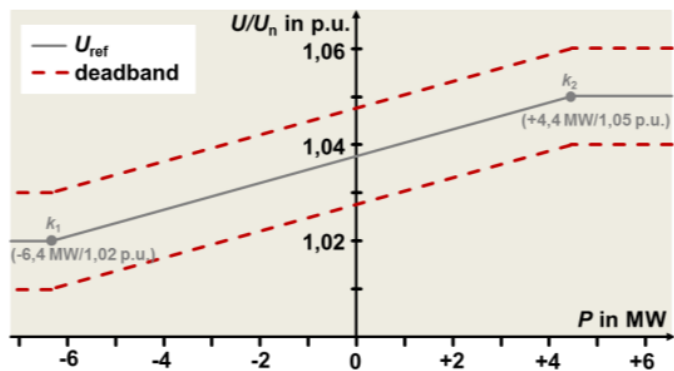

Figure 3: Profile of a compounding curve

The two break-points " $k_{1}$ " and " $k_{2}$ " define the design of the $U_{\text {ref }} / P_{\text {load }}$-curve. The break-point " $k_{2}$ " sets the upper limit of the reference voltage $U_{\text {ref }}$ in times of maximum voltage drops along the lines (high load, low generation). Break-point " $k_{1}$ " marks the lowest limit of the reference voltage $U_{\text {ref }}$ in times of a high reverse power flow to the overlaid HV-network which occurs in the hours of low demand with maximum feed-in of DERs. The profile is determined by calculating extreme scenarios (chapter 3 ). A higher gradient of the profile allows a more precise autotracking of the voltage, but it increases the number of tap changes as well, which reduces the service life of an OLTC without improving the utilisation of the available voltage band. The same applies for small deadbands. For the following simulations the deadband is set to $\pm 1,0 \% U_{\mathrm{n}}$.

\section{Advanced compounding by $Q$-measurement}

The basic idea behind the measurement of reactive power at the HV/MV-transformer is the identification of different situations with nearly no active power exchange between MV- and HV-grids. A slightly positive or negative active power flow means a more or less balanced ratio between demand and generation at that moment. The additional measurement of reactive power can help to differentiate between situations of high load and simultaneous high generation or low generation and low load. If the reactive power flow is relatively high, situations with high load and high generation can be expected due to the fact that most of the loads in the MV-level have an inductive character. Inductive behaviour applies in Germany also for DERs, which are operated by the DSOs with a reactive power support according to the BDEW-guideline. To counteract voltage rises occurring by a supply of active power, the DERs increase their reactive power demand linked with the active power supply up to a power factor $\cos \varphi$ of 0,95 . A typical reactive power curve applied by German DSOs is shown in figure 4.

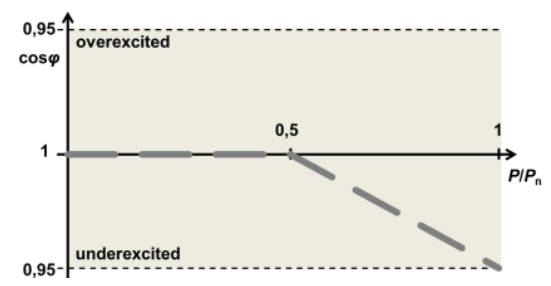

Figure 4: Typical $\cos \varphi(P)$-curve of DERs in Germany 
The target of the additional $Q$-measurement is to reduce the power losses in a network. In times of low or medium demand and low generation voltage deviations along the feeders are normally not at the maximum and free voltage margins are left. If these situations are identified the free margin can be used to operate the network at a slightly higher voltage level. For this purpose, the profile of the compounding curve has to be adjusted within the interval around no active power exchange with the HV-grid.

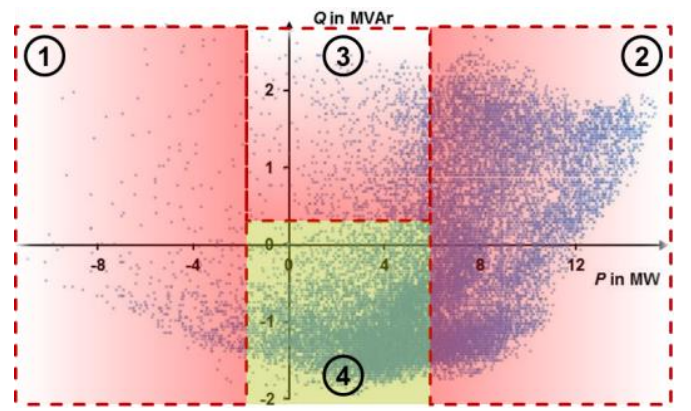

Figure 5: Quarter-hourly $P \cdot Q$-values of a $20-\mathrm{kV}$-network [16]

In figure 5 the $P$ - $Q$-balance of a rural $20-\mathrm{kV}$-network in Germany with a high rate of overhead lines is shown. In area ' 1 ' there is a high active power flow into the HVnetwork because of maximum feed-in of DERs and low load. High voltage rises occur along the lines and no further increase of the reference voltage $U_{\text {ref }}$ is possible compared to the control curve of a static compounding. In times of maximum consumption and minimum generation (area '2') $U_{\text {ref }}$ at the main MV-busbar is already at its maximum. To keep the voltage limits no further increase is possible. Times of low active power exchange between $\mathrm{HV}$ - and MV-network and high reactive power demand (area ' 3 ') belong to an almost balanced ratio of high load and feed-in with probably high voltage deviations in some lines of the network. A modification of the compounding curve would lead to violations of the voltage band. Only in the green area ' 4 ' the measurement of the reactive power exchange allows a slight increase of the voltage level to reduce power losses. These $P-Q$-values belong to times of low generation and low load. The high density shows that these situations often occur, especially in networks with mainly PV-units. In chapter 4 the effects on power losses, if an advanced compounding strategy by $Q$-measurements is used, are shown.

\section{Network topology for control methods}

As explained before, the usability of control methods for an OLTC strongly depends on the network topology. By the help of different generic networks it can be easily analysed for which topology the use of a control method allows improved voltage maintenance. On basis of eight simple generic 10-kV-networks (figure 6) it is analysed which of the methods presented in chapter 2 leads to an improved utilisation of the permitted voltage band. The line length of each feeder of the generic networks is determined to be $8 \mathrm{~km}$. A big icon for a generation unit or load represents an installed power of $3 \mathrm{MW}$. Small icons illustrate $1 \mathrm{MW}$. Four grids $(1-4)$ have a homogeneous character of the feeders considering points of common coupling and ratio between load and generation. Networks $5-8$ are more or less heterogeneous.

For each generic grid seven representative scenarios are simulated to analyse which topologies suit to the different control methods. These are two extreme scenarios (max. feed-in of DERs/no load and max. load/no generation) and the variation of load and generation in between in $25 \%$ steps. By the help of these scenarios almost all critical voltage deviations can be determined.
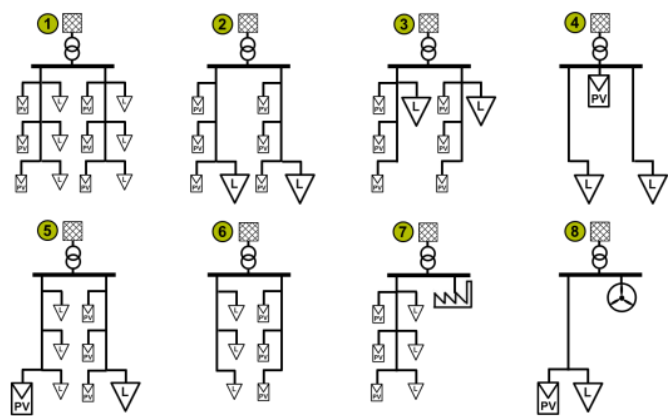

Figure 6: Topology of the eight generic networks

In figure 7 the results for network 5 having a PV-system with a high installed power at the end of one feeder and a huge load at the end of the other one are shown. In such a topology a compounding strategy or the use of a WAVC is able to achieve significant improvements for reducing the voltage deviations compared to a fixed reference voltage $U_{\text {ref }}=103,5 \% U_{\mathrm{n}}$. With a bandwidth of $9,1 \% U_{\mathrm{n}}$ between maximum and minimum voltage, the control method with fixed set point needs almost the complete available voltage band of $10 \%$ for the MV-level. A compounding is able to reduce the voltage deviation to $\Delta u=5,0 \% U_{\mathrm{n}}$ that means that more than $4 \%$ margin are gained for the installation of further DERs. WAVC achieves the same improvements.

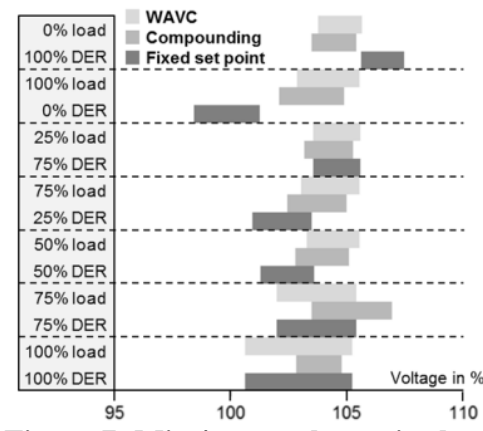

Figure 7: Min./max. voltages in the generic network 5

In general it can be said that in all homogenous networks (1-4) the use of compounding or WAVC decreases the voltage deviations. A big generation unit or load connected directly to the busbar (networks 4, 7 and 8) influences the active power flow into the $\mathrm{HV}$-grid without changing the voltage deviations in the feeders. Therefore, this additional active power flow distorts the correct estimation of voltage values when using a compounding strategy. However, if the distortion can be clearly allocated to a DER or load a compounding is still able to achieve almost as good results as a WAVC (network 4). This is not the case in networks having different types of generation or loads connected directly to the busbar. In this case a compounding can only scarcely estimate critical voltages in the feeders. In some special network topologies a compounding can even have negative effects on maintenance of the voltage level [10]. In these topologies the installation of measurement devices for WAVC is necessary to bring significant improvements. But there can be topologies where also the implementation of a WAVC does not have any positive effect. In network topologies having a long line where only loads are located and another one with DERs, none of the presented method for voltage management affects any improvement. 


\section{Results in a real MV-network}

The applicability of the different control methods and the effects on voltage maintenance and power losses are now demonstrated by use of power system simulations in a real $10-\mathrm{kV}$-network in quarter-hourly intervals over one year. A transformer with a rated power of $40 \mathrm{MVA}$ is feeding the network. It is equipped with an OLTC which is able to switch \pm 9 tap positions with a step size of $1,5 \%$ of $U_{\mathrm{n}}$. The network has a rural character with feeders up to almost $30 \mathrm{~km}$ line length (figure 8 ). The ratio of load/generation $(0,2$ to 2,7$)$ and the length of the lines vary significantly. The network contains several LV-networks with mainly households as loads. Industries are primarily connected directly to the MV-level. The maximum load of the grid can reach $P_{\mathrm{L}}=13,7 \mathrm{MW}$. The total installed power of DERs is $P_{\mathrm{G}}=13,5 \mathrm{MW}(10,5 \mathrm{MW} \mathrm{PV}$; 3,0 MW wind). For the simulations the load profile of typical households $\mathrm{H} 0$ and industries G0 are used [17]. The feed-in of the PV-units and wind turbines is based on real data of solar radiation and wind speed. All PV-units operate with reactive power support according to figure 4 . The basis for the comparison of the effects of the different methods is the control of the OLTC with a fixed set point. By setting the reference voltage $U_{\text {ref }}$ to a fixed value of $1,035 U_{\mathrm{n}}$ and the deadband to $\pm 1 \% U_{\mathrm{n}}$ it is possible to keep the permitted MV-limits $\left(U_{\min }>0,97 U_{\mathrm{n}} ; U_{\max }<1,07 U_{\mathrm{n}}\right)$ in all scenarios during a year, but there is no further voltage margin left.

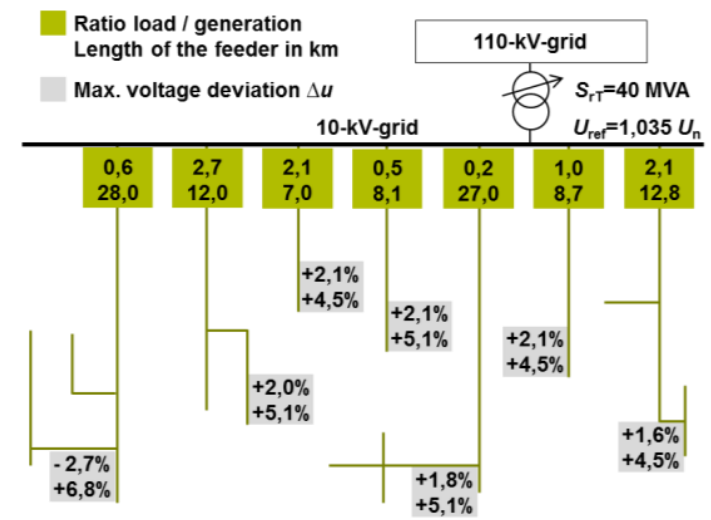

Figure 8: Power system simulation of a real $10-\mathrm{kV}$-network: Voltage deviations with fixed set-point control

During the annual simulations with a fixed set point a maximum active power flow of 9,6 MW and a maximum reverse flow of $-5,1 \mathrm{MW}$ occurs. Both values are used to determine the break-points of the compounding curve. Afterwards the profile has been optimised by simulating extreme scenarios. The break-points of the compounding curve are finally set to $k_{1}\left(-6,4 \mathrm{MW} / 1,02\right.$ p.u.) and $k_{2}(+4,4$ MW/1,05 p.u.). The deadband is kept constant to $\pm 1 \% U_{\mathrm{n}}$.

However, as explained in chapter 2, a static compounding curve does not always set the optimal reference voltage regarding power losses in a network. A measured active power flow close to $0 \mathrm{MW}$ can mean that load and feed-in are either high or low, whereas in times of low load and low feed-in the grid could be operated at a slightly higher voltage level. In figure 9 the green area marks the interval where an advanced compounding with $Q$-measurement improves network operation by setting a higher reference voltage. In those simulations $U_{\text {ref }}$ has been increased by $\Delta u=1 \% U_{\mathrm{n}}$ for all measurements with an active power flow between $-1 \mathrm{MW}$ to $4 \mathrm{MW}$ and a reactive power flow of $0 \mathrm{MV}$ ar to 5 MVar. A higher reduction of power losses can be achieved by a finer subdivision of the interval for the advanced compounding. For instance, in times of a very low load and low generation (interval with reactive power $0 \leq \Delta Q \leq 2 \mathrm{MV}$ ar) the voltage level could be increased by $\Delta u=2 \% U_{\mathrm{n}}$ without any violation of the voltage limit.

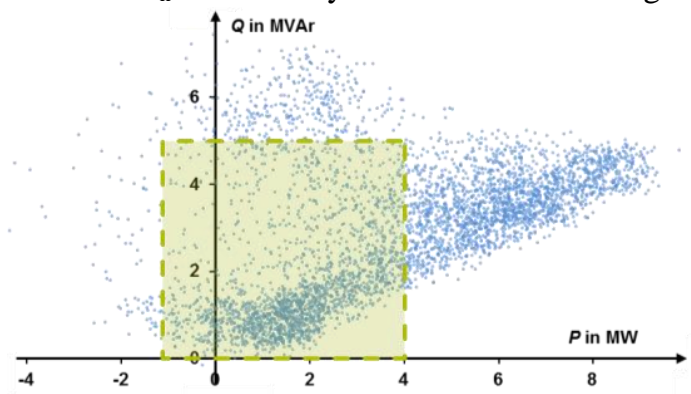

Figure 9: Advanced compounding in the 10-kV-network

In the analysed grid the maximum and minimum voltage values occur at six different nodes in four feeders. Thus, for perfect implementation of a WAVC the installation of at least six measuring devices is necessary. A tap change at the OLTC is triggered when the maximum voltage at one of the critical nodes exceeds 1,07 $U_{\mathrm{n}}$ or drops below $0,995 U_{\mathrm{n}}$. Because of the exact knowledge of the voltage deviations at all critical nodes, a WAVC allows the most efficient utilisation of the voltage band and therefore also a reduction of power losses. Whereas the decrease of power losses is low (only 3,5\% compared to a fixed reference voltage) the benefits for voltage deviations are remarkable (table 2). The reduction of the required voltage margin for the MV-network from $\Delta u=9,4 \% U_{\mathrm{n}}$ to $7,4 \% U_{\mathrm{n}}$ allows almost a doubling of the installed capacity of DERs in the MV-level, if no thermal limitations occur.

Tab. 2: Comparison of control methods for a 10-kV-network

\begin{tabular}{|l|c|c|c|c|c|c|}
\cline { 2 - 7 } \multicolumn{1}{c|}{} & $\begin{array}{c}\boldsymbol{u}_{\max } \\
\text { p.u. }\end{array}$ & $\begin{array}{c}\boldsymbol{u}_{\min } \\
\text { p.u. }\end{array}$ & $\begin{array}{c}\Delta \boldsymbol{u} \\
\text { p.u. }\end{array}$ & $\begin{array}{c}\boldsymbol{u}_{\text {average }} \\
\text { p.u. }\end{array}$ & $\begin{array}{c}\text { Taps/ } \\
\text { day }\end{array}$ & $\begin{array}{c}\boldsymbol{W}_{\text {losses }} \\
\mathbf{M W h}\end{array}$ \\
\hline $\begin{array}{l}\text { Fixed } \\
\text { set point }\end{array}$ & 1,068 & 0,974 & 0,094 & 1,022 & 2,0 & 356,9 \\
\hline $\begin{array}{l}\text { Static } \\
\text { Compounding }\end{array}$ & 1,063 & 0,983 & 0,080 & 1,032 & 3,4 & 350,3 \\
\hline $\begin{array}{l}\text { Advanced } \\
\text { Compounding }\end{array}$ & 1,064 & 0,992 & 0,072 & 1,037 & 4,0 & 347,5 \\
\hline WAVC & 1,069 & 0,995 & 0,074 & 1,043 & 0,7 & 344,3 \\
\hline
\end{tabular}

The results verify that for network topologies similar to the analysed MV-network, costs for measurement devices and ICT-infrastructure can be avoided by controlling the OLTC by a compounding strategy. For the utilisation of the voltage band, an optimised compounding strategy has almost as good effects as a WAVC. The required voltage margin is reduced by $1,4 \%$ of $U_{\mathrm{n}}$ compared to a reference voltage of $1,035 U_{\mathrm{n}}$ at the busbar. The wear of the OLTC is expected to be higher because of the increasing number of tap changes. But even if the use of the compounding strategy doubles the tap changes per day, the number is still in a tolerable level with an average of four per day [5]. One peculiarity in the analysed $10-\mathrm{kV}$-network has to be mentioned. The voltage values in figure 8 show that there is one feeder which has much higher deviations $(-2,7 \%)$ $+6,8 \%)$ than all the other feeders. That implies that the hosting capacity for DERs of the total network could be further increased by an enhancement of the lines or the installation of innovative grid equipment like e.g. a boost transformer [18] in this particular feeder. 


\section{Conclusion and outlook}

If the installation of new DERs in a distribution network is limited by voltage problems and not by thermal overload of grid equipment, innovative methods for the control of the OLTC of the HV/MV-transformer are an economic solution to increase the hosting capacity for many network topologies. Since the active power flow to the HV-network is already measured, the use of a compounding strategy is a very cost-efficient method to improve the utilisation of the permitted voltage band. This method works accurate in networks having feeders with a more or less similar length of line and same ratio of generation and load. In topologies with big generation units or loads connected directly or close to the main busbar the use of a compounding strategy is limited. Different types of generation units (PV, wind power, biogas) or loads (households, industry) constrain the applicability of a compounding strategy as well. In these topologies the more expensive WAVC, for which measurement devices and ICT-infrastructure are necessary, might be the only control method which is able to avoid voltage band violation. If the distribution of generation and demand is extremely heterogeneous between the different feeders of a network, neither a compounding strategy nor a WAVC are able to reduce voltage deviations compared to a fixed set point voltage for the OLTC.

To reduce the losses in a network static compounding can be upgraded to an advanced compounding strategy by an additional usage of the reactive power flow through the transformer as an input value for the profile of the control curve. By the help of a $Q$-measurement, the times of high generation can be identified if the DERs are operated with typical reactive power support profiles according to the technical guidelines in Germany. This allows to slightly increase the voltage level of the network in times of low voltage deviations along the lines. Even if these off-peak situations occur relatively frequently during a year, the benefit is limited because of the quadratic dependency of the power losses from the current. The implementation of optimised control methods for the OLTC of an HV/MVtransformer normally increases the number of tap changes. Therefore, to find a cost-efficient solution against voltage band problems possible additional maintenance or shorter life-times because of a higher number of tap changes always have to be considered.

In further studies other additional control variables like e.g. the usage of local solar radiation for estimation of the feed-in of big photovoltaic systems, wind measurements or load forecasts should be analysed [15]. Furthermore, the use of current flows in single feeders, which are often measured anyway in a substation, could be considered to improve the effects of a compounding strategy and raise the applicability for different network topologies further.

Finally, it can be said: The implementation of advanced control methods for OLTCs in MV-networks cannot avoid network expansion caused by thermal overload. But this paper has shown that these methods are able to reduce the extent of needed network expansions and can increase the hosting capacity for DERs in different network topologies in case of limitations by the voltage band. Compared to a compounding strategy, a WAVC allows a better utilisation of the voltage band in more kinds of topologies with the drawback of a possibly lower availability and higher costs.

\section{References}

[1] Bundesverband der Energie- und Wasserwirtschaft e.V.: "Erneuerbare Energien und das EEG: Zahlen, Fakten, Grafiken", Berlin (2014).

[2] Bundesverband der Energie- und Wasserwirtschaft e.V.: "Erzeugungsanlagen am Mittelspannungsnetz - Richtlinie für den Anschluss und Parallelbetrieb von Erzeugungsanlagen am Mittelspannungsnetz", Berlin (2008).

[3] Verband Elektrotechnik Elektronik Informationstechnik e.V.: VDE-AR-N 4105: "Erzeugungsanlagen am Niederspannungsnetz - Technische Mindestanforderungen für Anschluss und Parallelbetrieb von Erzeugungsanlagen am Niederspannungsnetz", Berlin (2011).

[4] Talavera, Ignacio; et al.: "Voltage regulation through wide area voltage control of HV/MV transformers and Inverters" reactive power support", ICREPQ, Cordoba (2014).

[5] Krämer, Axel: "On-Load Tap-Changers for Power Transformers - Operation Principles, Applications and Selection", Verlag Maschinenfabrik Reinhausen GmbH, Regensburg (2000).

[6] Leiße, Ingmar: "Efficient Integration of Distributed Generation in Electricity Distribution Networks", Lund University - Dissertation, Lund (2013).

[7] DIN EN 50160: "Characteristics of voltage in public power supply systems", DKE, Berlin (2011).

[8] Stetz, Thomas; et al.: "Methods for Maintaining Voltage Limitations in Medium Voltage Systems", ETG-Congress, Würzburg (2011).

[9] Lakervi, E.; Holmes, E.J. "Electricity distribution network design", Institution of Electrical Engineers, London (2003).

[10] Piga, Erika; Geschiere, Alex: "Optimisation of the voltage regulation in the dutch $M V$-grid", CIRED, Turin (2005).

[11] Gajic, Zoran; Aganovic, Samir: "Advanced tap changer control to counteract power system voltage stability", $\mathrm{ABB}$ $\mathrm{AB}$, Vasteras (2006).

[12] A. Eberle GmbH \& Co. KG: "Spannungsregler REG-D", Manual - A. Eberle, Nürnberg (2009).

[13] Körner, Christian; et al.: "Gaining experience with a regulated distribution transformer", CIRED Workshop, Lisbon (2012).

[14] Werner, Manfred; et al.: "Optimisation of $H V / M V$ transformer-voltage-control in distribution networks with a high proportion of distributed generation", CIRED Workshop, Lisbon (2012).

[15] Dropalla, Roland; et al.: "Comparison of concepts to avoid grid expansion in medium voltage distribution network", ETG-Congress, Berlin (2013).

[16] Talavera, Ignacio; et al.: "Flexible reactive power exchange between distribution and transmission networks: Case study", CIRED Conference, Lyon (2015).

[17] Bundesverband der Energie- und Wasserwirtschaft e.V. (BDEW): "VDEW Standard Load Profiles", www.bdew.de, Berlin (2014).

[18] Tomlinson, Males; et. al.: "Series-Stacked Medium Voltage Electronic Voltage Regulator", ECCE - Energy Conversion Congress and Exposition, Phoenix (2011). 